\title{
Approach towards Establishing a Backup Satellite Attitude Control System Based on the Photovoltaic Array
}

\author{
Andry V. Sedel'nikov ${ }^{1}$, Sergey L. Safronov ${ }^{1}$, Alexander S. Filippov ${ }^{1}$ and Ekaterina K. Savich ${ }^{2}$ \\ Samara National Research University/Department of space engineering, Samara, Russia \\ Samara National Research University/Department of aircraft production and quality management in mechanical engineering, Samara, \\ Russia
}

\begin{abstract}
During the design stage of a spacecraft it is important to carry out a thorough analysis of potential onboard equipment failures in order to develop means of their mitigation so that the satellite would remain fully functional for the entirety of its lifespan. The article demonstrates an idea towards establishing a backup satellite attitude control system based on the photovoltaic array of the power control system.
\end{abstract}

\section{Introduction}

Space environment makes a significant impact on spacecraft's components. For example, during the solar flares the intensity of the Sun wind could increase by several orders of magnitude. This could potentially lead to failures or incorrect operation of onboard electronics. Operation of a spacecraft with failed equipment could lead to a significant performance decrease up to a total loss of functionality.

\section{Problem definition}

An attitude control system breakdown is an example of a failure that significantly impairs satellite's performance. Incorrect operation of attitude control effectors usually is non-reparable in space conditions and can lead to the loss of control of spacecraft's orientation. However, these type of failures are not very common.

Maintaining correct operation of satellite's onboard measuring instruments is another problem caused by the conditions of the space environment [1]. Primary information is formed based on the measurement data in order to generate the control moment of the satellite's effectors. Incorrect operation of onboard measuring instruments in the mode of compensation of microaccelerations on the experimental unit of the "Aist" small satellite did not allow the magnetic effectors of scientific equipment "MAGKOM" to reduce the angular velocity of rotation of the spacecraft to the target values. Moreover, as the authors of [2] point out, the control moment in some cases increased rather than reduced the angular velocity of the "Aist" small satellite.
In the event of a failure in the attitude control system of the onboard control complex, usually, spacecraft transitions into an undirected flight for the time necessary for the automated elimination of this failure by utilizing hardware (switching between onboard equipment sets, structural adjustment, etc.) and functional reserves (return to stable operating modes, establishing a new program of operation of on-board equipment that would be able to work around the failure, etc.). Generally, redundancy with backup hardware allows to restore the working capacity of the spacecraft in most cases. However, in a number of situations, for example, when all backup sets fail, this approach is ineffective. If the standard methods of performance restoration are unavailable or ineffective, the satellite might enter a critical state, for example, dangerous battery discharge levels, leading to the complete loss of functionality.

Creation of on-board software, able to integrate the functions of instruments and systems, allows to increase survivability and extend the life of the spacecraft by redistributing functions between operational equipment and implementing new logic of work [3].

Thus, the search for new solutions for providing means of functional redundancy of the on-board equipment, their implementation at the satellite's design stage and implementation on board at the stage of regular operation will increase the likelihood of successful operation of the spacecraft and potentially extend its lifespan.

In this paper, the implementation of the backup satellite attitude control system based on the use of the photovoltaic array of the power supply system is presented. 
Current, A

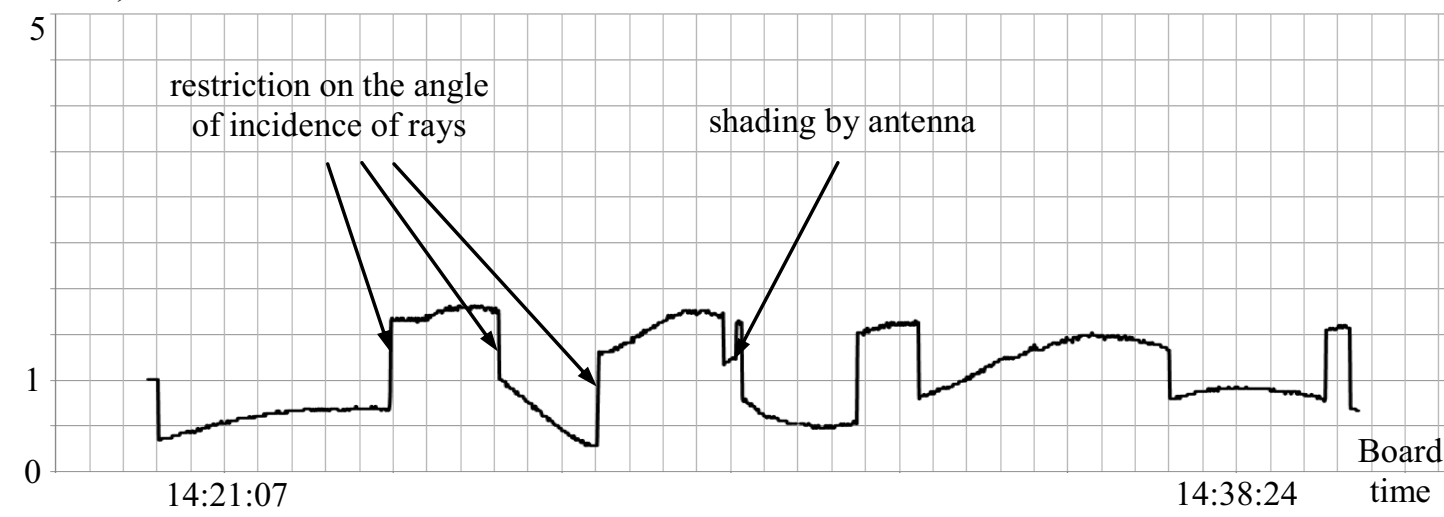

Current, A

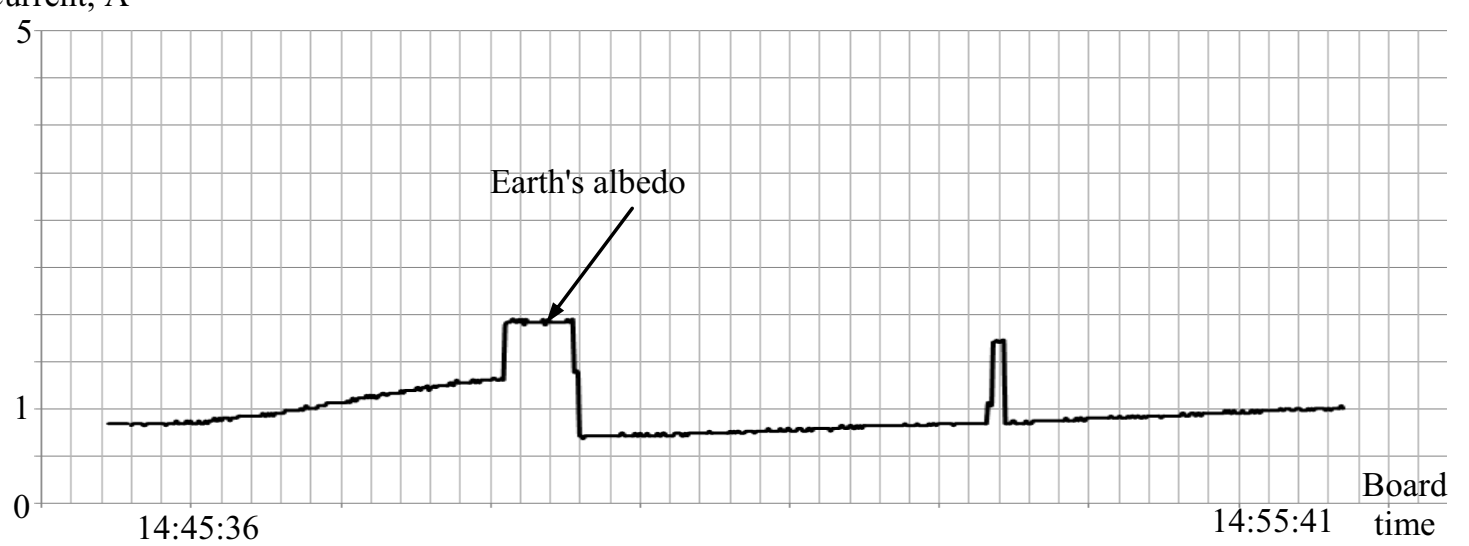

Figure 1. The current produced as a function of time

\section{Attitude estimation based on the solar elements' elecric current values for a small satellite}

The problem of satellite's attitude control using data on the electric current values from the photovoltaic arrays has been considered before. For example, the paper [4] describes a similar problem. However, the satellite considered in that paper had its photovoltaic array mounted on foldable panels. Since these panels are naturally oscillating during the satellite's operation [5], the problem, described in [4] looks to be more complex.

The paper [6] considered a similar problem. However, it only took into account the data from a single panel, that was processed using the Kalman filtering. Another similar approach was discussed in [7]. Only four out of six outer panels of the nanosatellite were covered with solar cells and the spacecraft was stabilized by rotation around the longitudinal axis, imposing certain limitations on the attitude control algorithm. It is also worth noticing, that for a satellite with six side panels a situation, when a significant charge current flows from all six faces due to the Earth's albedo is not uncommon. The problem of correct data analysis in this situation was considered in [8].

\section{Algorithm for evaluation of rotary motion of a small satellite}

"Aist" small satellite has a solar battery. Photovoltaic cells are glued to five of the six panels of the body. In addition, there is a separate solar panel, which is installed on top of the sixth panel of satellite. The solar battery is made on the basis of three-stage arsenide-gallium photovoltaics.

The satellite does not have a system of orientation and stabilization. Its shape is close to the cube, and the center of mass is in the vicinity of the geometric center of the cube. The satellite makes a non-oriented flight and rotates at an angular velocity in the range from 0.3 to $1.3 \%$.

The photovoltaic array of the "Aist" small satellite produces electrical current proportional to the cosine of the angle of incidence of the Sun's rays from the normal to the active surface. The first approximation of the value of the current's strength will be:

$$
i_{\text {cur }}=i_{\mathrm{sc}} \cdot \cos \alpha .
$$

where $i_{s c}$ - current value of the solar cell of the corresponding type ( $\mathrm{Si}, \mathrm{GaAs}, \mathrm{CdTe}$, etc.).

The figure 1 shows graphs of the current produced as a function of time.

The position of each solar cell is defined in the base coordinate system and is described by a unit vector $n_{s c i}$ coinciding with the normal to its operating surface.

The satellite control system records telemetry information with a specified frequency including the electric current values from solar cells. Knowing $i_{c u r}$ it is possible to define a conical surface, whose generatrix contains the vector of direction to the Sun R. If $\alpha_{i} \geq \pi / 2$ the electric current value from the solar cell would be zero. 
In general, three of the six faces of the outer surface of the satellite are facing the Sun. In other words, three normals to the surfaces of photovoltaic arrays located on different faces of the satellite form sharp angles with the direction toward the Sun. They can be estimated using equation (1). These angles can be related to the Euler angles between the bound and heliocentric coordinate systems, assuming that the spacecraft is a material point:

$$
\left\{\begin{array}{l}
\sin \varepsilon_{1}=\sqrt{2-\cos \alpha_{1}-\cos \alpha_{2}-\cos \alpha_{3}} \\
\sin \varepsilon_{2}=\sqrt{1-\cos \alpha_{1}-\cos \alpha_{2}+\cos \alpha_{3}} \\
\sin \varepsilon_{3}=\sqrt{1-\cos \alpha_{1}+\cos \alpha_{2}-\cos \alpha_{3}}
\end{array}\right.
$$

We are using the normalization condition as a verification for the estimation of the Euler angles using system (equation (2)), which, taking equation (2) into account, will take the following form:

$$
3 \cos \alpha_{1}+\cos \alpha_{2}-\cos \alpha_{3}=2
$$

Equation (3) can serve as a criterion for the admissibility of application of equation (1) for estimating the angles between the normals to the working surfaces of photoconverters and the direction to the Sun.

The position of the coupled coordinate system relative to the geocentric orbital coordinate system can be found by using the corresponding rotation matrices from the heliocentric first to the fixed geocentric, and then to the geocentric orbital coordinate system.

The model should take the following factors into account:

1. the position of the satellite in the geocentric coordinate system and the orbital parameters at each moment of time should be known with the required accuracy (can be ensured by processing signals of the GLONASS and GPS systems with the necessary frequency);

2. the direction to the Sun must be calculated constantly with the required accuracy, based on the data on the position of the satellite in orbit and the time of year;

3 . the currents from the solar panels, placed on not more than three adjacent faces of the satellite, should be taken into account in the orientation calculation, based on the knowledge of the direction vector on the Sun in the associated coordinate system,

4. the photoconverters should not be shaded by external elements of the satellite;

5. the Earth's albedo;

6. the solar battery produces electric current at an angle of incidence of sunlight on its surface more than 150 .

In the figure 1 cases of shading of the solar battery by external antennas (factor 4), the effect on the current value of the earth's albedo (factor 4 ), as well as the illustration of factor 6 are noted.

\section{Numerical simulation for the flight unit of the "Aist"small satellite}

With the purpose of approbation of the possibility of using the proposed algorithm in practice, we evaluate its performance on the example of the flight unit of the "Aist" small satellite (figure 2).

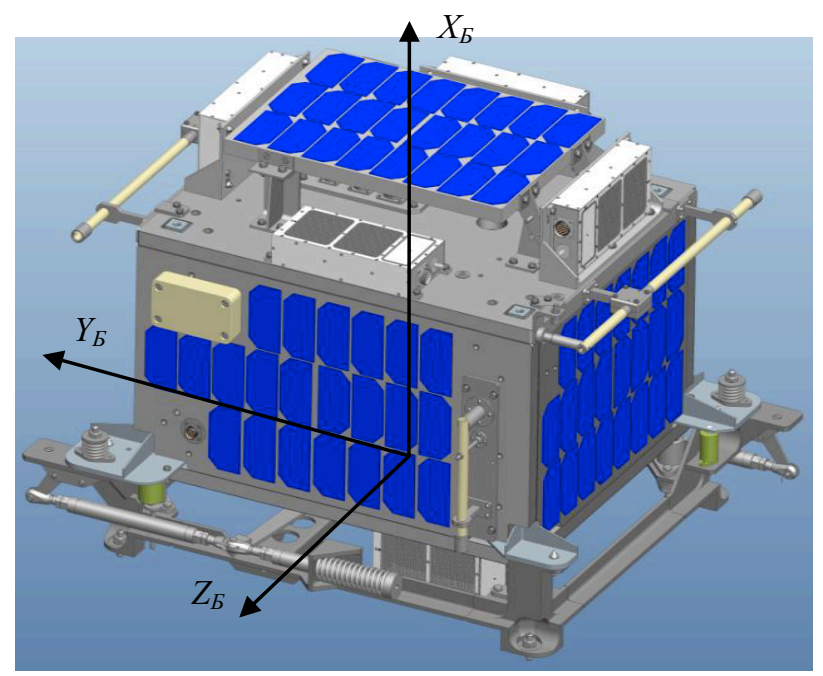

Figure 2. External layout of the "AIST" small satellite

The "Aist" small satellite was put into orbit on 21.04.2013 by its separation from the spacecraft "BionM" No. 1, operating in a near-circular orbit with height of about $570 \mathrm{~km}$ and inclination of $64.9 \mathrm{o}$. Its mass, together with the separation device, was $53 \mathrm{~kg}$. The "Aist" did not have a full-fledged attitude control system for orbital motion, but it was capable to reduce its rotation velocity around the center of mass due to the interaction of electromagnets with the magnetic field of the Earth [5]. Electromagnets were part of the MAGKOM scientific equipment (figure 3) and provided the control moment for reducing the angular velocity of rotation of the satellite.

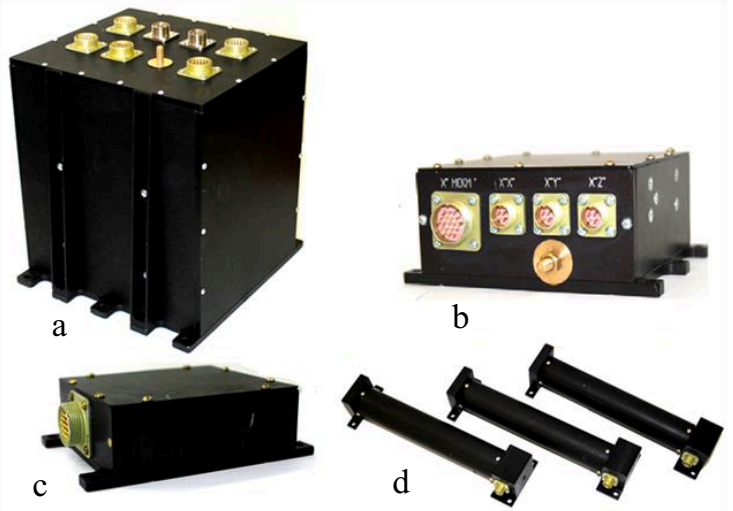

Figure 3. MAGCOM scientific equipment: a - central control unit; b electromagnet control unit; $\mathrm{c}$ - three-component magnetometer; $\mathrm{d}-$ electromagnets.

Electromagnets were switched on twice during the operation period of the flight unit of the "Aist" small satellite. In both cases they were able to reduce the angular velocity of its rotation. 
The measurements of the Earth's magnetic field, obtained using onboard magnetometers of the MAGKOM scientific equipment (figure 4), were used as primary information for the control of electromagnets. Since the electromagnets on the flight unit of the "Aist" small satellite had worked as intended, the recorded data of measurements of the Earth's magnetic field can be considered correct within the scope of the paper. These measurements will serve as a benchmark for assessing the possibility of determining the parameters of the rotational motion of the satellite, using data on the electric current values from solar cells.

It should be noted, that in telemetric information the electric current values from the solar cells were recorded with a measurement interval of $1 \mathrm{~min}$. Therefore, in this paper we are not able to make estimations of the accuracy of determining the parameters of rotational motion, but only about the principle possibility of such an estimate. The results of estimating the average value of the angular velocity modulus of the flight unit of the "Aist" small satellite with the use of magnetometers and electric current values from solar cells on May $10^{\text {th }}, 2013$ are shown in figure 3 . The average value of the angular velocity vector was estimated by the following formula:

$$
|\overrightarrow{\vec{\omega}}|=\frac{\sqrt{\left(\varepsilon_{10}-\varepsilon_{11}\right)^{2}+\left(\varepsilon_{20}-\varepsilon_{21}\right)^{2}+\left(\varepsilon_{30}-\varepsilon_{31}\right)^{2}}}{\Delta t} .
$$

In equation (4) $\varepsilon_{10}, \varepsilon_{20}, \varepsilon_{30}$ and $\varepsilon_{11}, \varepsilon_{21}, \varepsilon_{31}$ - are estimates of the Euler angles (equation (2)) between the coupled and heliocentric coordinate systems at time instants, separated by an interval $\Delta t$.

Magnetometer measurements were recorded at the $\Delta t_{1}=6 \mathrm{~s}$ intervals. This is why the estimate of the average value of the angular velocity modulus using magnetometer data was carried out using the maximum likelihood method [9]:

$$
|\overline{\vec{\omega}}|=\frac{1}{n} \sum_{i=1}^{n}\left|\vec{\omega}_{i}\right|
$$

In equation (5):

$$
\begin{gathered}
\left|\vec{\omega}_{i}\right|=\frac{1}{\Delta t_{1}} \sqrt{K^{2}+L^{2}+M^{2}} \\
K=\arccos \left(\frac{B_{x i}}{\left|\vec{B}_{i}\right|}\right)-\arccos \left(\frac{B_{x i-1}}{\left|\vec{B}_{i-1}\right|}\right) \\
L=\arccos \left(\frac{B_{y i}}{\left|\vec{B}_{i}\right|}\right)-\arccos \left(\frac{B_{y i-1}}{\left|\vec{B}_{i-1}\right|}\right) \\
M=\arccos \left(\frac{B_{z i}}{\left|\vec{B}_{i}\right|}\right) \arccos \left(\frac{B_{z i-1}}{\left|\vec{B}_{i-1}\right|}\right)
\end{gathered}
$$

$$
\vec{B}_{i-1}\left(B_{x i-1} ; B_{y i-1} ; B_{z i-1}\right) \quad \text { and } \quad \vec{B}_{i}\left(B_{x i} ; B_{y i} ; B_{z i}\right) \quad-
$$

measurements of the components of the vector of induction of the Earth's magnetic field by magnetometers, separated by a time interval $\Delta t_{1}$;

$\mathrm{n}$ - number of measurements taken over the $\Delta t$ time interval.

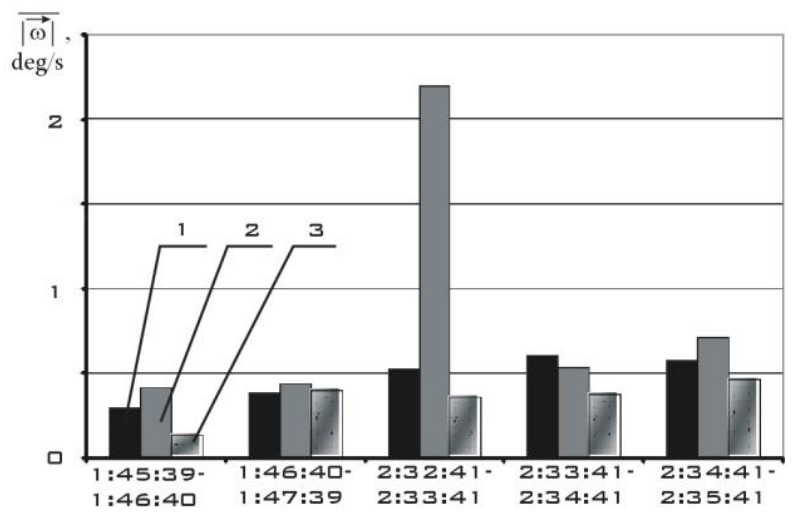

Figure 4. The results of estimating the average value of the angular velocity modulus using: 1 - the first magnetometer sensor; 2 - the second magnetometer sensor; 3 - using the proposed algorithm

As can be seen from the presented results, the differences between the estimates of the angular velocity of rotation of the small satellite by two different magnetometers are more significant than between the magnetometers and the proposed algorithm. This clearly demonstrates the possibility of correct estimation of the parameters of the rotational motion of a small spacecraft using electric current data from solar cells and the proposed evaluation algorithm.

To improve the accuracy of the estimation of rotational motion parameters, the proposed algorithm should measure the current from solar cells no less than once in two seconds.

If the spacecraft has solar cells mounted on deployable panels, then for correct use of the proposed algorithm it is necessary to place photoconverters not only on the panels themselves, but also on different faces of the external surface of the spacecraft, since the proposed algorithm requires knowing the orientation of the three vectors of the normal to the faces of the spacecraft relative to the Sun.

\section{Conclusion}

It is possible to estimate the parameters of the rotational motion of a spacecraft that has its outer surface covered with photoconverters. Using data on the electric current values from solar cells.

The proposed algorithm can be used both by itself and together with others. The combination of two methods of orientation on one satellite (as a primary and backup), taking into account specific recommendations for switching from one orientation mode to another and back, requires the development and implementation of special software, but it also provides an additional positive effect - increase of the survivability of the satellite. 


\section{Acknowledgment}

The study was carried out at the expense of the grant of the Russian Science Foundation (Agreement No. 17-7910456).

\section{References}

1. A. V. Sedelnikov, "Fast analysis of onboard measurements of the earth magnetic field for the purpose of microaccelerations decrement on board of the "AIST" small spacecraft during its uncontrolled orbital flight", International Review of Aerospace Engineering, vol. 11, № 2, pp. 160-166, (2018).

2. A. N. Kirilin, E. V. Shakhmatov, V. A. Soifer, R. N. Akhmetov, S. I. Tkachenko, A. B. Prokofev, et. al., "Small satellites "AIST" constellation - design, construction and program of scientific and technological experiments", Procedia Engineering, vol. 104, pp. 43-49, (2015).

3. R. N. Akhmetov, V. P. Makarov, A. V. Sollogub, "Bypass principles of automated spacecraft survivability control", in Proc. 23rd Saint Petersburg International Conference on Integrated Navigation Systems (ICINS 2016), St. Petersburg, pp. 501-505, (2016)
4. A.A. Davydov, Determination of parameters of attitude motion of a small communication satellite using the data of measurements of current of solar panels, Cosmic Research, Vol. 49, № 4, P. 335-344, (2011)

5. A. V. Sedelnikov, A. A. Serpukhova, "Simulation of a flexible spacecraft motion to evaluate microaccelerations", Russian Aeronautics, vol. 52, № 4, pp. 484-487, (2009).

6. E.B. Babcock CubeSat attitude determination via Kalman filtering of magnetometer and solar sell data, 25th Annual AIAA/USU Conference on Small Satellites, p. 1-10 (2011).

7. R. Burton, S. Rock, J. Springmann, J. Cutler Online attitude determination of a passively magnetically stabilized spacecraft, Acta Astronautica, Vol. 133, P. 269-281,( 2017)

8. A.V. Sedelnikov Mean of Microaccelerations Estimate in the Small Spacecraft Internal Environment with the Use of Fuzzy Sets, Microgravity Science and Technology, https://doi.org/10.1007/s12217-018-9620-y, pp.1-7, (2018)

9. G. P. Anshakov, A. I. Belousov, A. V. Sedelnikov The problem of estimating microaccelerations aboard Foton-M4 spacecraft, Russian Aeronautics, vol. 60 , № 1 , pp. 83 - 89, (2017). 